\title{
An Empirical Study of the Maximum Degree of Undersampling in Compressed Sensing for $T_{2}^{*}$-weighted MRI
}

\author{
Carole Lazarus $^{\mathrm{a}, \mathrm{b}, \mathrm{c}}$, Pierre Weiss ${ }^{\mathrm{d}, \mathrm{e}}$, Alexandre Vignaud ${ }^{\mathrm{a}, \mathrm{b}}$, Philippe \\ $\mathrm{Ciuciu}^{* \mathrm{a}, \mathrm{b}, \mathrm{c}}$ \\ ${ }^{a}$ NeuroSpin, CEA Saclay, 91191 Gif-sur-Yvette cedex, France \\ ${ }^{b}$ Université Paris-Saclay, France \\ ${ }^{c}$ Parietal, INRIA, 91120 Palaiseau, France \\ ${ }^{d}$ ITAV USR3505 CNRS, 31000 Toulouse, France \\ e IMT UMR 5219 CNRS, 31400 Toulouse, France
}

\begin{abstract}
Magnetic Resonance Imaging (MRI) is one of the most dynamic and safe imaging modalities used in clinical routine today. Yet, one major limitation to this technique resides in its long acquisition times. Over the last decade, Compressed Sensing (CS) has been increasingly used to address this issue and offers to shorten MR scans by reconstructing images from undersampled Fourier data. Nevertheless, a quantitative guide on the degree of acceleration applicable to a given acquisition scenario is still lacking today, leading in practice to a trialand-error approach in the selection of the appropriate undersampling factor. In this study, we shortly point out the existing theoretical sampling results in CS and their limitations which motivate the focus of this work: an empirical and quantitative analysis of the maximum degree of undersampling allowed by CS in the specific context of $T_{2}^{*}$-weighted MRI. We make use of a generic method based on retrospective undersampling to quantitatively deduce the maximum acceleration factor $R_{\max }$ which preserves a desired image quality as a function of the image resolution and the available signal-to-noise ratio (SNR). Our results quantify how larger acceleration factors can be applied to higher resolution
\end{abstract}

\footnotetext{
${ }^{*}$ Corresponding author

Email address: philippe.ciuciu@cea.fr (Philippe Ciuciu*)
} 
images as long as a minimum SNR is guaranteed. In practice however, the maximum acceleration factor for a given resolution appears to be constrained by the available SNR inherent to the considered acquisition. Our analysis enables to take this a priori knowledge into account, allowing to derive a sequence-specific maximum acceleration factor adapted to the intrinsic SNR of any MR pipeline. These results obtained on an analytical $T_{2}^{*}$-weighted phantom image were corroborated by prospective experiments performed on MR data collected with radial trajectories on a 7 Tesla scanner with the same contrast. The proposed framework allows to study other sequence weightings and therefore better optimize sequences when accelerated using CS.

Keywords: Compressed Sensing, MRI, image quality, maximum undersampling factor.

\section{Introduction}

Reducing the acquisition time in Magnetic Resonance Imaging (MRI) has been a major direction of research in recent years. Speeding up image acquisition while maintaining diagnostic quality is indeed crucial in many respects.

5 The improvement of patient comfort together with a reduced risk of motion artifacts are examples of consequential advantages. Moreover, in dynamic imaging for instance, accelerated acquisitions are critical to visualize rapid physiological changes. Finally, the resulting gain in time can be invested in increasing the spatial and temporal resolution or in supplementary scans. In this context, considerable effort has been spent on developing methods to accelerate data acquisition while preserving image quality. One recent and most promising strategy is the Compressed Sensing (CS) theory which consists in reducing the number of measurements and thus the acquisition time (TA) by exploiting the compressibility of MR images [1, 2]. Using CS, data can be massively undersampled by a given acceleration factor $R$ compared to the fully-sampled Cartesian acquisition, while ensuring conditions for optimal image recovery. Examples of recent successful applications of CS in MRI are numerous 3 especially in 
two-dimensional (2D) or three-dimensional (3D) dynamic MRI [4, 5]. In cardiac imaging for instance, CS made it possible to perform single breath-hold and even free-breathing 2D cine MRI while including motion-correction 6, 7. Moreover, combining CS with golden-angle radial trajectories, the XD-GRASP method allows free-breathing cardiac cine imaging and 3D dynamic contrastenhanced MRI of the liver [8, 9].

While many efforts have been made to improve the CS methodology in MRI both on the acquisition [10, 11, 12, 13, 14, 15, 16, 17, 18, and the reconstruction [1, 19, 20, 21, 22] sides, the question on the actual limitations to the acceleration rate in CS for a given experimental setup has been hardly addressed. Hence, CS could considerably benefit from a study analyzing in a quantitative way the major practical limitations to the degree of acceleration in CSMRI. 30 Such work would provide CS users with valuable information to design their MR sequence by selecting the appropriate acceleration factor adapted to each scan instead of using a trial-and-error approach. This is however a delicate question as numerous parameters affect the quality of CS reconstructions including the image size and the available signal-to-noise ratio (SNR) for a particular acquisition [23. In this work, we will offer an insight into the following question:

In CSMRI, how should the maximum acceleration factor be selected as a function of the image size and the available SNR, in order to maintain a desired image quality?

40

One may expect that existing theoretical sampling results would provide an analytical answer to this question. Unfortunately, if the existing literature proposes a rather comprehensive qualitative analysis of CSMRI, the constants involved in the theorems and concentration inequalities are often crudely estimated. The 45 result is that most practically implemented sampling schemes should be considered as mere heuristics.

Following this observation which we briefly elaborate in a first theoretical part (Section 2), we propose to conduct an empirical analysis of the consid- 
ered preceding question in the case of $T_{2}^{*}$-weighted imaging. Applying a generic method based on retrospective undersampling (Section 3), we present its results (Section 4) on an analytical image for two types of MR sampling schemes and a conventional $\ell^{1}$-based non-linear reconstruction. The image quality dynamics could thus be quantitatively analyzed and threshold values were identified. The maximum acceleration factor $R_{\max }$ was also quantitatively determined as a function of the image size and the available input SNR to reach a desired image quality. Moreover, we present a retrospective and prospective experimental validation of these results based on $T_{2}^{*}$-weighted images acquired with a 7 Tesla scanner on an ex vivo baboon brain. We finally propose a method to deduce a sequence-specific maximum undersampling factor circumscribed by the intrinsic SNR of any given acquisition.

\section{The theory of CS in MRI and its limitations}

The theory of compressed sensing is often considered a mature field by nonspecialists. While this belief proves to be quite accurate for Gaussian measurements, many important questions are still open when dealing with structured measurements and structured signal recovery, as met in MRI. In this section, we review a few major theoretical results and open questions to motivate our experimental study.

\subsection{The case of unstructured measurements}

Let

$$
\sigma_{s}(\mathbf{z})=\min _{\mathbf{z}^{\prime} \in \mathbb{C}^{n}, \mathrm{~s}-\text { sparse }}\left\|\mathbf{z}-\mathbf{z}^{\prime}\right\|_{1}
$$

denote the $\ell^{1}$-tail of a vector $\mathbf{z} \in \mathbb{C}^{n}$. This function is often used to characterize 70 the compressibility of a signal $\mathbf{z}$.

The following theorem [24, Theorem 9.13] provides an accurate description of the recovery guarantees in the case of unstructured Gaussian measurements.

Theorem 1. Assume that $\mathbf{A} \in \mathbb{C}^{m \times n}$ is a matrix with i.i.d. random Gaussian components. There exist universal constants $C_{1}, C_{2}, D_{1}$ and $D_{2}$ such that, for 
any $1 \leq s \leq n$, for any $\epsilon \in(0,1)$, if

$$
m \geq C_{1} s(\ln (n / 2)+1)+C_{2} \ln (2 / \epsilon),
$$

then, with probability at least $1-\epsilon$, for all vectors $\mathbf{z} \in \mathbb{C}^{n}$, given the measurements $\mathbf{y}=\mathbf{A z}+\mathbf{e} \in \mathbb{C}^{m}$, where $\mathbf{e}$ is a measurement error satisfying $\|\mathbf{e}\|_{2}^{2} \leq m \sigma^{2}$, we get:

$$
\|\mathbf{z}-\hat{\mathbf{z}}\|_{2} \leq D_{1} \frac{\sigma_{s}(\mathbf{z})}{\sqrt{s}}+D_{2} \sqrt{m} \sigma
$$

where

$$
\hat{\mathbf{z}}=\underset{\left\|\mathbf{A} \mathbf{z}^{\prime}-\mathbf{y}\right\|_{2} \leq \sqrt{m} \sigma}{\arg \min }\left\|\mathbf{z}^{\prime}\right\|_{1}
$$

The value of this theorem lies in the fact that it provides a good understanding of the reconstruction quality with respect to the signal's compressibil75 ity (captured by $\sigma_{s}(\mathbf{z})$ ) and the input SNR (captured by $\sigma$ ). In particular, it shows that if $s \mapsto \frac{\sigma_{s}(\mathbf{z})}{\sqrt{s}}$ decreases sufficiently fast with $s$, a small number of measurements will be sufficient to reconstruct the true signal, up to an error proportional to $\|e\|_{2}$. The $\ell^{1}$-tail $\sigma_{s}$ explains the role of resolution in the theory of CS. Fig. 1 shows the evolution of the normalized $\sigma_{s}(\mathbf{z})$ with respect to the normalized sparsity $s / n$ for the phantom image Fig. 2A) at different resolutions ( $n=128 \times 128, n=512 \times 512$ and $n=2048 \times 2048)$. Two observations can be drawn from this graph: on the one hand, the larger the relative sparsity of $\mathbf{z}$, the smaller its $\ell^{1}$-tail $\sigma_{s}(\mathbf{z})$. On the other hand and more importantly, the higher the resolution, the faster the $\ell^{1}$-tail decay. Hence, CS will allow using higher sub-sampling factors at higher resolution for an equal error. Similar phenomena are explained in more details in 25].

Let us now describe the limitations of this theorem. First, it does not capture the denoising capabilities of $\ell^{1}$ reconstructions. The term $\sqrt{m} \sigma$ in (3) coincides with the amount of noise in the data and increases with the number of measure90 ments $m$. In practice it is often observed that $\ell^{1}$ minimization not only allows to recover missing information, but also serves as a regularizer able to denoise the data. Second, the constants $C_{1}, C_{2}, D_{1}$ and $D_{2}$ are not tight and can be huge in some variants of this theorem, meaning that the lower bound on the 


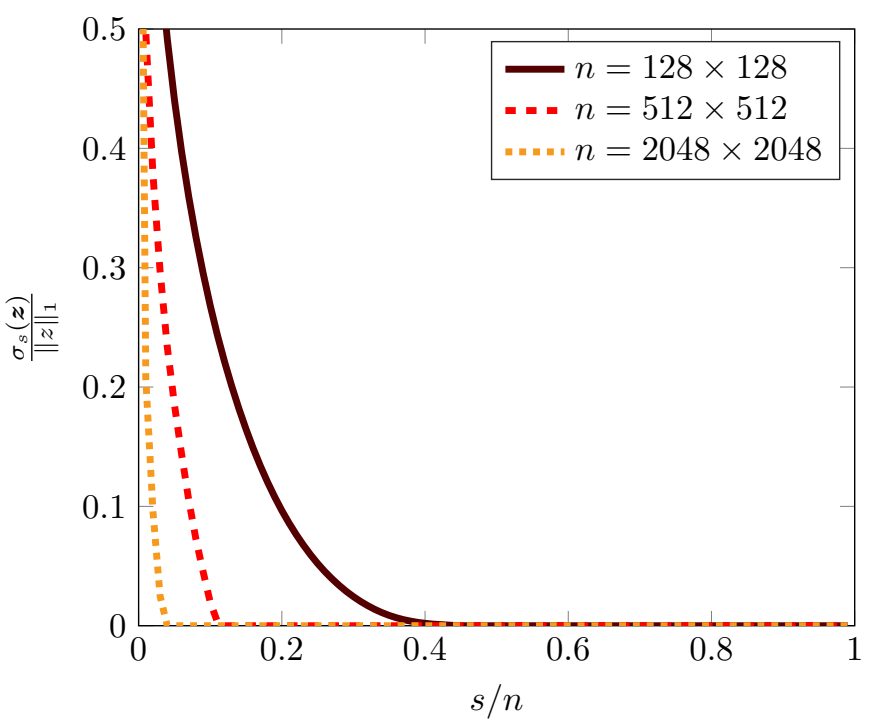

Figure 1: Normalized $\ell^{1}$-tail $\sigma_{s}(\mathbf{z})=\min _{\mathbf{z}^{\prime} \in \mathbb{C}^{n} \text {, s-sparse }}\left\|\mathbf{z}-\mathbf{z}^{\prime}\right\|_{1}$ with respect to the relative sparsity $s / n$ for different resolutions of the brain phantom image in Fig. 2 2 A): $n=128 \times 128$, $n=512 \times 512$ and $n=2048 \times 2048$. The $\ell^{1}$-tail decays faster at higher resolutions, thus allowing the use of larger undersampling factors for higher resolutions in the context of CS.

number of measurements $m$ may actually be quite large, or equivalently that the undersampling factor $R=n / m$ may be close to 1 or even smaller than 1 .

This last criticism has to be moderated by the theoretical analysis of phase transitions in 26]. It is now well known that in the case of noiseless measurements, perfect recovery will occur with high probability whenever $m \geq$ $2 s(\log (n / s)+1)+\epsilon$ and will fail with high probability when $m \leq 2 s(\log (n / s)+$ 1) $-\epsilon$, where $\epsilon$ is a small margin. This shows that the minimum number of measurements for good recovery is very well understood in this case.

\subsection{The case of structured measurements and structured signals}

In MRI, signals are highly structured: all brain images share strong similarities and can be modeled much more precisely than arbitrary $s$-sparse signals. A simple model to describe this structure is the sparsity by levels in wavelet bases [12]: each wavelet sub-band of the image contains a number of nonzero coefficients bounded by a known quantity at each scale. Moreover, the traditional 
way of acquiring data in MRI is far from independent Gaussian measurements: Fourier transform values of the image are probed along continuous trajectories.

The current sampling theory in this challenging setting can safely be described as significantly less comprehensive than the case of Gaussian measurements. Let us however remark that significant advances were proposed recently [12, 25, 27, 15, 28. Without introducing the theorems, let us detail the main conclusions and limitations of these studies. One important result argues that low frequencies should be probed more often than high frequencies. The reason is subtle and not just due to the fact that there is more information in the low frequencies [? ]. To understand the reconstruction limits, one needs to precisely describe the links between wavelet bases, Fourier bases and sparse by levels signals. The current major limitations can be listed as follow:

- The constants appearing in the theorems are usually far too large to be of any practical use. This is due to inaccurate proof techniques, but it is currently unknown how much the constants can be lowered. Establishing phase transition results for structured sampling schemes as in the case of Gaussian measurements still requires significant mathematical advances.

- Designing optimal sampling densities is still an open problem. Theorems provide quantitative upper-bounds on the number of measurements necessary for perfect reconstruction, which allows designing decent densities. However, proving optimality results requires to establish tight lower bounds. In practice the design of good sampling densities is therefore heuristic.

- The noise is not correctly handled. The stability to noise and compressibility is established, but the dependencies are far less optimistic than those in Theorem 1

- Most results are only available for orthogonal wavelet transforms and $\ell^{1}$ reconstructions. It is now admitted that much better reconstruction results can be obtained in practice by using redundant transforms, learned 
dictionaries or non-convex regularizers. The understanding of CS in this setting is still very partial.

- In the case of measurements collected along curves, very little is known. Let us mention that the case of parallel lines as proposed in [1] was analyzed completely in 28 .

Overall, we see that existing theoretical results provide a good qualitative analysis of CS, allowing to guide the design of sampling and reconstruction schemes. However, to date, theorems are unable to provide quantitative conclusions on the number of needed measurements in CSMRI.

\section{An empirical and quantitative study}

While being theoretically inextricable, the proposed question can be empirically addressed and its answer may provide valuable practical and quantitative information to CSMRI. Hence, we proposed the following methodology.

\subsection{A generic method}

In order to empirically quantify the effects of the image size and input SNR on the reconstructed image quality from undersampled data, we propose the following generic framework. All notations are listed in (Table 1).

First, a database of $2 \mathrm{D}$ reconstructions is constituted for a large range of image sizes, input SNR and acceleration factors. Square images are considered and characterized by their image size, denoted by $N \in \mathbb{N}$, which refers to the dimension of the corresponding $N \times N$ square matrix. An image of size $N \in \mathcal{N}$, where $\mathcal{N}$ is the studied range of sizes, is therefore composed of $N^{2}$ pixels. Images of different input SNR $\left(\mathbf{x}_{N}^{\mathrm{SNR}}\right)$ were obtained by adding complex-valued zero-mean white Gaussian noise of varying standard deviation $\sigma \in \Sigma$ to the full complex $k$-space data of a noiseless image $\left(\mathbf{x}_{N}^{\infty}\right)$, where $\Sigma$ is the studied range of noise levels. Each noise level $(\sigma \in \Sigma)$ was then expressed as its corresponding input $S N R \in \mathcal{S N} \mathcal{R}$, a quantity more commonly used in the MRI community, where $\mathcal{S N \mathcal { R }}$ denotes the studied range of SNR. The input SNR is computed 
Table 1: Notations used throughout the paper.

\begin{tabular}{|c|c|}
\hline Notations & Meaning \\
\hline$N \in \mathcal{N}$ & Image size \\
$m$ & Number of measurements \\
$R=N^{2} / m \in \mathcal{R}$ & Undersampling factor \\
$\sigma \in \Sigma$ & Noise level \\
$\mathrm{SNR} \in \mathcal{S} \mathcal{N} \mathcal{R}$ & Signal-to-noise ratio \\
$\mathbf{x}_{N} \in \mathbb{C}^{N^{2}}$ & Image of size $N$ \\
$\mathbf{x}_{N}^{\infty}$ & Noiseless image of size $N$ \\
$\mathbf{x}_{N}^{\mathrm{SNR}}$ & Noisy image of size $N$ characterized by its input SNR \\
$\widehat{\mathbf{x}}_{R}$ & Reconstructed image from $R$-fold undersampled data \\
$Q ; Q_{0}$ & Image quality metric ; image quality threshold \\
$R_{\max }\left(Q_{0}, N, \mathrm{SNR}\right)$ & Maximum undersampling factor \\
$\mathbf{z} \in \mathbb{C}^{N^{2} \times q}$ & q-fold undecimated wavelet representation of image $\mathbf{x}$ \\
$\mathbf{y} \in \mathbb{C}^{m}$ & Measured Fourier data \\
$\mathbf{F}_{\Omega}$ & Fourier Transform over the support $\Omega \subseteq\left\{1, \ldots, N^{2}\right\}$ \\
$\mathbf{\Psi}$ & q-fold undecimated wavelet transform $(L=4, q=3 L+1)$ \\
\hline
\end{tabular}
of the mean signal in a region-of-interest (ROI) in the white matter (smaller orange circle in Fig. 24)) over the standard deviation in the background signal (larger yellow circle in Fig. 2A)). Finally, a set of undersampling schemes and image reconstruction is specified as input of the proposed pipeline 1 . The undersampling or acceleration factor $R \in \mathcal{R}$ is given by $R=N^{2} / m$, where $m$ is the number of measurements and $\mathcal{R}$ the studied range of undersampling factors.

Once the database is complete, it is possible to introduce the concept of a maximum acceleration factor $R_{\max }\left(Q_{0}, N, \mathrm{SNR}\right)$ as the function of (i) the targeted image quality expressed in terms of a quality threshold $Q_{0}$ for a given image quality metric $Q$, (ii) the image size $N$ and (iii) the image input SNR:

$$
R_{\max }\left(Q_{0}, N, \mathrm{SNR}\right)=\underset{R}{\arg \max }\left\{Q\left(\widehat{\mathbf{x}}_{R}, \mathbf{x}_{N}^{\mathrm{SNR}}\right) \geqslant Q_{0}\right\}
$$

where $\mathbf{x}_{N}^{\mathrm{SNR}}$ is the reference image with given image size $N$ and SNR and $\widehat{\mathbf{x}}_{R}$ the corresponding reconstruction retrospectively undersampled by a factor $R$. Similar threshold over image quality score was already used in 29] for diagnostic 
Pipeline 1 Find $R_{\max }\left(Q_{0}, N\right.$, SNR $)$

Input: An undersampling method undersample and the associated reconstruction reconstruct.

Input: Image quality metric $Q$ and a threshold $Q_{0}$

1: for $\mathrm{N}$ in $\mathcal{N}$ do

2: $\quad$ Take an image $\mathbf{x}_{N}^{\infty}$ of size $\mathrm{N}$

3: $\quad$ for $\sigma$ in $\Sigma$ do

4: $\quad \mathbf{x}_{N}^{\mathrm{SNR}} \leftarrow \mathbf{F F T}{ }^{-1}\left(\mathbf{F F T}\left(\mathbf{x}_{N}^{\infty}\right)+\mathcal{N}(0, \sigma)\right)$.

5: $\quad$ for $\mathrm{R}$ in $\mathcal{R}$ do

6: $\quad$ data $\leftarrow$ undersample $\left(\mathbf{x}_{N}^{\mathrm{SNR}}, R\right)$

7: $\quad \widehat{\mathbf{x}}_{R} \leftarrow$ reconstruct $($ data $)$

8: $\quad$ end for

9: $\quad$ end for

10: end for

Output: $R_{\max }\left(Q_{0}, N, \mathrm{SNR}\right) \forall \mathrm{N} \in \mathcal{N}, \mathrm{SNR} \in \mathcal{S N \mathcal { R }}$

175 utility

Finally, to study separately the effect of either the image size or the noise level, one of these parameters was kept constant while the other was varied.

\subsection{The studied pipeline}

In this work, we applied the above method to both analytical and experimental data in the case of $T_{2}^{*}$-weighted imaging.

\subsection{1. $2 D$ images}

The studied images shown in Fig. 2A-B) are derived from the analytical brain phantom with a contrast similar to $T_{2}^{*}$-weighting, which was introduced by Guerquin-Kern et al [30]. Considered image sizes belonged to the set $\mathcal{N}=$ $185\{128,256,512,1024,2048\}$. 


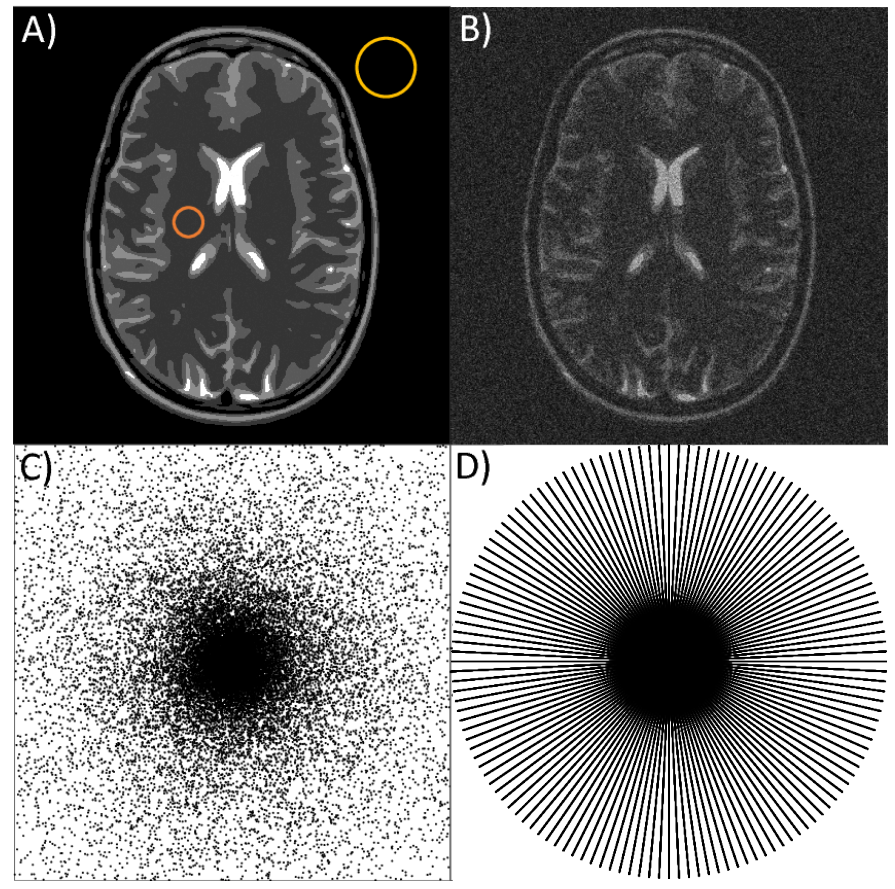

Figure 2: Guerquin-Kern analytical phantom of a brain image [30] for an image size of $N=512$ with highest studied SNR of 110 (A) and lowest input SNR of 3 (B). The smaller orange and larger yellow circles respectively represent the ROI in the white matter and the area in the background signal used to calculate the input SNR. Examples of iid sampling schemes of Fourier space along the chosen variable density is displayed for $N=512$ and $R=10$ in (C) and symmetric isotropic radial trajectory for $N=1024$ and $R=15$ in (D).

\subsubsection{Input $S N R$}

We studied a set of input SNR ranging from 3 to 110. Resulting noisy images are displayed in Fig. 2A)-B) for the most extreme SNR values.

\subsubsection{Undersampling schemes}

Two undersampling schemes were considered in this work: identically and independently distributed (iid) and radial samplings. First, non-Cartesian samples were iid randomly drawn according to polynomially decaying distribution of degree 2, with a plateau in the low frequencies, which provided good reconstruction performance across the different image sizes [31. An example of the 

tive Soft Thresholding Algorithm [34]) was implemented to solve the minimization problem (5). Finally, the regularization parameter was tuned over a range 
Table 2: Correspondence between SSIM scores and Mean Opinion Scores (MOS) between an image and a reference 39 .

\begin{tabular}{|c|c|c|c|}
\hline SSIM & MOS & Quality & Impairement \\
\hline$\geq 0.99$ & 5 & Excellent & Imperceptible \\
{$[0.95,0.99)$} & 4 & Good & Perceptible but not annoying \\
{$[0.88,0.95)$} & 3 & Fair & Slightly annoying \\
{$[0.5,0.88)$} & 2 & Poor & Annoying \\
$\leq 0.5$ & 1 & Bad & Very annoying \\
\hline
\end{tabular}

of $\left[10^{-6} ; 10^{-2}\right]$ so as to select the reconstruction of best quality according to the

criterion introduced below.

\subsubsection{Image quality metrics}

To quantitatively assess the image quality, we chose to work with two referencebased image quality metrics: the structural similarity (SSIM) index introduced in 35 and the Normalized Root Mean Square Error (NRMSE in \%). On the one

${ }_{225}$ hand, the SSIM attempts to model the human visual system (HVS) and is increasingly employed in the MRI community to assess image quality [36, 37, 38, and on the other hand the NRMSE is a commonly used intensity-based metric calculated as follows (Eq. 6):

$$
\operatorname{NRMSE}(\widehat{\mathbf{x}}, \mathbf{x})=\frac{\|\widehat{\mathbf{x}}-\mathbf{x}\|_{2}}{\|\mathbf{x}\|_{2}}
$$

where $\widehat{\mathbf{x}}$ is the reconstructed image from Eq. (5) and $\mathbf{x}$ the reference. While the $\operatorname{SSIM}$ index, $\operatorname{SSIM}(\widehat{\mathbf{x}}, \mathbf{x})$, varies between 0 (null correspondence with the reference) and 1 (perfect match with the reference), the NRMSE is comprised between a $0 \%$ and $100 \%$ error. For each image size, the corresponding reference was chosen as the fully sampled Cartesian image of same size presenting a SNR of 110, which is the highest studied SNR in this work. Moreover, these indexes were computed over cropped images deprived of their background with a binary mask. Since both metrics presented very similar tendencies, NRMSE performance was only shown in the first part of the results and the SSIM index was used for the rest of the study. 
Table 3: Parameters of $T_{2}^{*}$-weighted GRE sequence used for experiments.

\begin{tabular}{|c|c|}
\hline Parameters & Values \\
\hline Repetition time (TR) & $60 \mathrm{~ms}$ \\
Echo time (TE) & $30 \mathrm{~ms}$ \\
Flip angle (FA) & $10^{\circ}$ \\
Slice thickness (SL) & $5 \mathrm{~mm}$ \\
Field of view (FOV) & $205 \mathrm{~mm}$ \\
Total NEX for $N=512$ & 15 \\
Total NEX for $N=1024$ & 82 \\
\hline
\end{tabular}

Furthermore, to quantitatively represent the targeted image quality, we selected an arbitrary SSIM threshold denoted by $Q_{0}$ whose value was set to 0.9 as this is considered to convey a fair image quality [39]. A correspondence between SSIM scores and Mean Opinion Scores (MOS) is displayed in Table 2.

\subsection{Experimental validation with MRI acquisitions}

MR acquisitions were performed on a 7 Tesla Siemens scanner (Siemens Healthineers, Erlangen, Germany), with a 1-channel transmit and 1-channel receive coil (InVivo Corp., Gainesville, FL, USA) to provide experimental noisy data for retrospective undersampling. An ex vivo baboon brain conserved in a fluorinert solution was imaged with a 2D $T_{2}^{*}$-weighted Gradient Recalled Echo (GRE) sequence. All animal studies were conducted in accordance with the European convention for animal care and the NIHs Guide for the Care and Use of Laboratory Animals. The acquisition was fully-sampled on the Cartesian grid for image sizes of $N=512$ and $N=1024$ and consisted of $N$ lines collecting $N$ Fourier samples (no oversampling). Parameters of the sequence are summarized in Table 3. To get a wide range of input SNR, data averaging over several measurements was performed in the Fourier domain. To reach an input SNR of 110, image sizes $N=512$ and $N=1024$ needed respectively a number of 15 and 82 averages, referred as to NEX (Number of EXcitations) hereafter. The resulting magnitude images of various input SNR were then retrospectively undersampled with the presented iid and radial sampling patterns. 
Moreover, prospective radial acquisitions were performed for an image size of $N=1024$ and a acceleration factor of $R=15$ using the trajectory displayed in Fig. 2D). Imaging parameters were the same as for the fully-sampled Cartesian acquisition at $N=1024$, including the NEX to vary the input SNR.

\section{Results}

Fig. 3AA)-B) respectively show the SSIM and NRMSE scores as a function of the input SNR for four increasing undersampling factors, under a constant image size of $N=1024$. For a given acceleration factor, as the input SNR gets larger the SSIM increases to an asymptotic value corresponding to the maximum score denoted by $\operatorname{SSIM}_{l i m}(N, R)$ (Fig. 3A). More precisely, the image quality reaches a plateau, as soon as the input SNR is sufficiently high. Hence, the concept of a minimum input SNR required to attain the asymptotic image quality score can be defined: once this SNR is reached, there is no gain in image quality anymore.

For the presented case $N=1024$ and $R \in\{5,10,20,30\}$, the values of $\operatorname{SSIM}_{l i m}(N, R)$ are reported in Table 4. As the undersampling factor grows, the corresponding asymptotic SSIM value drops. A similar but reversed trend can be observed on Fig. 3B) for the NRMSE index where a plateau is visible as well.

Table 4: Asymptotical SSIM values, $\operatorname{SSIM}_{l i m}(N=1024, R)$, for $R \in\{5,10,20,30\}$.

\begin{tabular}{|c|c|c|c|c|}
\hline$R$ & 5 & 10 & 20 & 30 \\
\hline SSIM $_{l i m}$ & 0.97 & 0.93 & 0.89 & 0.85 \\
\hline
\end{tabular}

\subsection{Influence of image size}

Fig. 4 shows the SSIM scores as a function of the image size for four undersampling factors, under a constant intermediate input SNR of 81. For a given acceleration factor, as the image size gets larger the SSIM index increases in a concave manner. Moreover, two regimes can be identified in the dynamics 

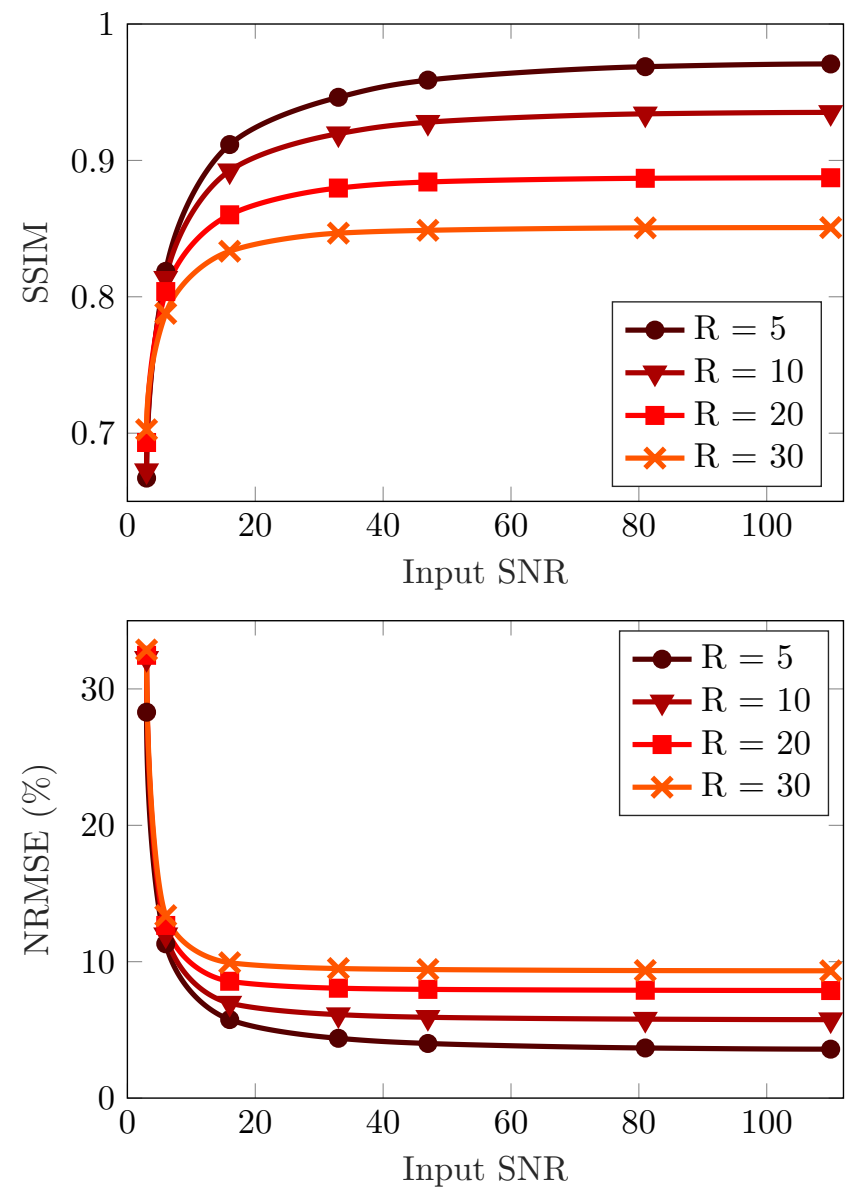

Figure 3: For a constant image size $N=1024$, evolution of A) SSIM scores and B) NRMSE scores as a function of input SNR, for different acceleration factors of 5, 10, 20 and 30, in the case of analytical images reconstructed from iid-undersampled data. For both metrics, as the input SNR increases, the image quality improves and approaches its limit. A stationary regime is reached for high input SNR, where SSIM and NRMSE scores are approximately equal to their maximum and minimum values respectively for a given acceleration factor.

of image quality scores: a high-resolution regime corresponding to large image sizes and a low-resolution regime corresponding to small image sizes (the FOV is kept constant). On the one hand, for large image sizes above $N=512$ the image quality scores are quasi invariant by change in image size and are close to their maximum value for each acceleration factor. On the other hand, for small 
image sizes the image quality rapidly drops to severely low scores.

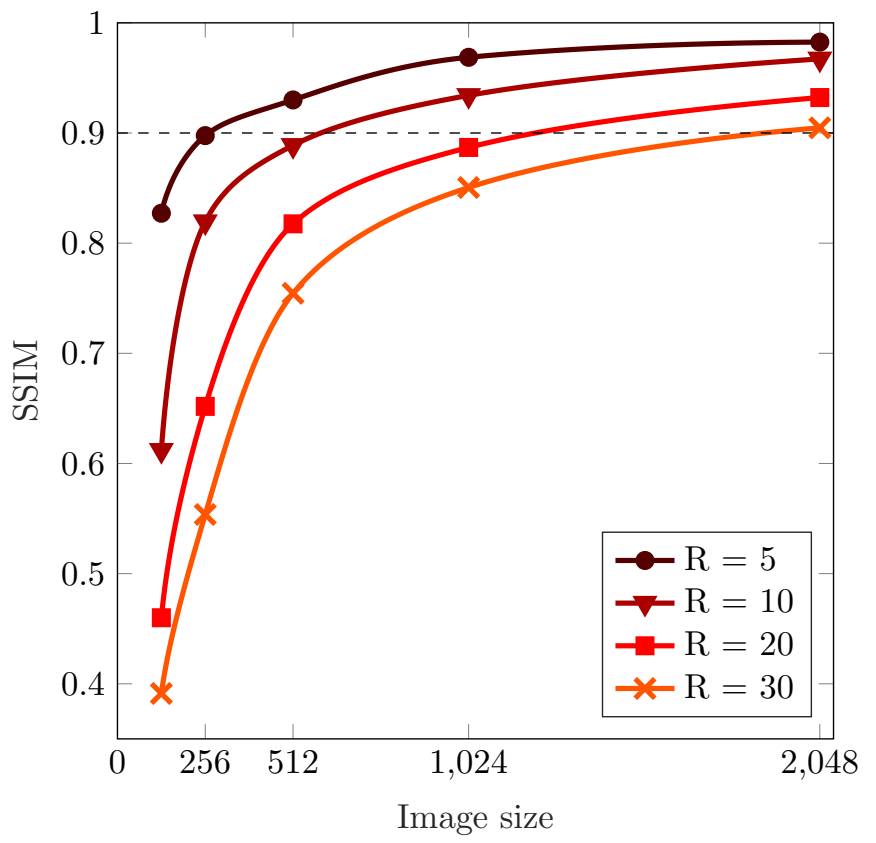

Figure 4: For a constant input SNR of 81 , evolution of SSIM scores as a function of image size, for different acceleration factors of 5, 10, 15 and 30, in the case of analytical images reconstructed from iid-undersampled data. SSIM scores increase in a concave manner as the image size gets larger. Two dynamics can be distinguished: a low-resolution regime where image quality rapidly diminishes as the image size decreases and a high resolution regime where image quality remains stable as the image size gets larger. The dashed black line represented the chosen quality threshold $Q_{0}=0.9$ and highlights the combinations $(N, R)$ of image sizes and acceleration factors which allow to maintain the targeted image quality.

Furthermore, Fig. 4allows to determine the combinations of image size and acceleration factor $(N, R)$ guaranteeing a SSIM threshold of $Q_{0}=0.9$ (dashed line) at the studied input SNR. While any undersampling factor up to $R=30$ can be applied to an image of size $N=2048$ and still ensure SSIM scores above 0.9 , an image size of $N=256$ cannot be accelerated more than 5 times in order to meet $Q_{0}=0.9$. 


\subsection{Maximum acceleration factor}

The previous results on image size and SNR influences can be combined to determine the maximum acceleration factor allowed in a given situation, $R_{\max }\left(Q_{0}, N, \mathrm{SNR}\right)$.

A 3D map of the maximum undersampling factor allowing SSIM scores higher than $Q_{0}=0.9$ is shown in Fig. 5 A) for the analytical phantom, as a function of image size $N$ and input SNR. The flat aspect of the surface along the input SNR direction conveys the image quality reaching the previously observed stationary state once a specific minimum SNR has been attained. In contrast, as can be seen on the 2D projection of the surface on Fig. $5 \mathrm{~B}$ ), the maximum acceleration factor keeps growing as the image size increases, reaching values of 5, 8, 16 and 30 for images sizes of 256, 512, 1024 and 2048 respectively. Moreover, the natural logarithmic scale on the image size direction used on this view indicates a super-linear growth rate of $R_{\max }$ as a function of image size.

\subsection{Acquisition intrinsic $S N R$ and $R_{\max }$}

We propose to further specify the aforementioned functional $R_{\max }$ defined on all the possible combinations of studied input SNR and image sizes, by taking into account the acquisition intrinsic SNR available in practice which depends on the image resolution and the considered MR sequence. Indeed, any acquisition is characterized by its sequence relaxation parameters (TR, TE, FA), resolution parameters $(N, F O V, S L)$, the MR scanner itself and its transmitting/receiving chain including the coils, and the imaged object relaxation properties $\left(T_{1}, T_{2}\right)$. Hence, for a particular acquisition and fixed FOV, there exists a unique relationship between the image size $N$ and the input SNR. Graphically, this $N$-SNR relation can be projected onto the $R_{\max }$ surface to delineate where the maximum undersampling factor of the sequence of interest lives in practice. Fig. 5 shows this experimental $N$-SNR level line (in orange) in the case of the presented GRE sequence (see Table 3) which was projected on the studied domain. The $2 \mathrm{D}$ view of this line is depicted in Fig. $5 \mathrm{~B}$ ) and illustrates 

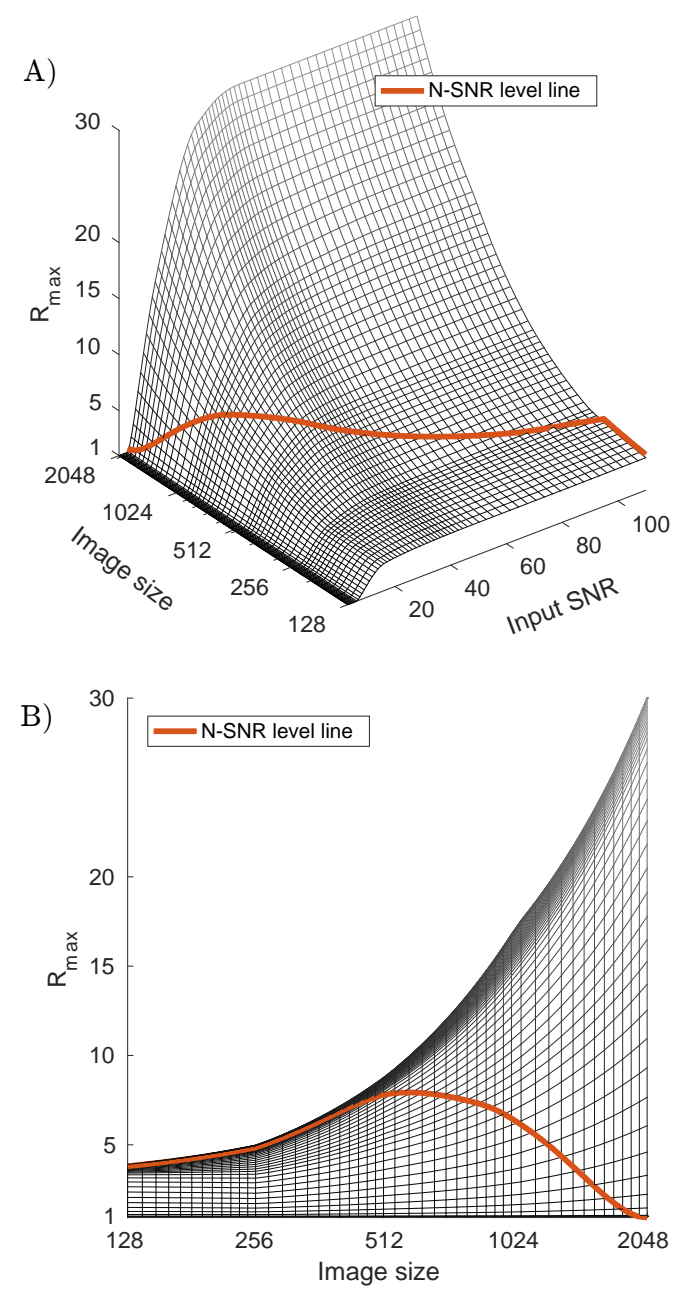

Figure 5: Analytical phantom $R_{\max }$ results in the case of iid undersampling. A) 3D view of the maximum undersampling factors allowing SSIM scores above $Q_{0}=0.9$ as a function of image size $N$ and input SNR. B) Its $2 \mathrm{D}$ projection on the $\left(R_{\max }, N\right)$ plane. Knowing the image size and the available input SNR of any acquisition, the corresponding maximum undersampling factor allowing to reach the targeted image quality $Q_{0}=0.9$ can thus be derived. The experimental $N-$ SNR level line (orange line) of the presented $T_{2}^{*}$-weighted GRE acquisition (see section III.C) was added on both graphs. It represents the projection onto the $R_{\max }$ surface of the relationship between the intrinsic SNR available in practice for this particular acquisition and the image size. For the considered sequence, only acceleration factors below the $N$ - SNR level line will thus meet or exceed the targeted image quality (see B). 


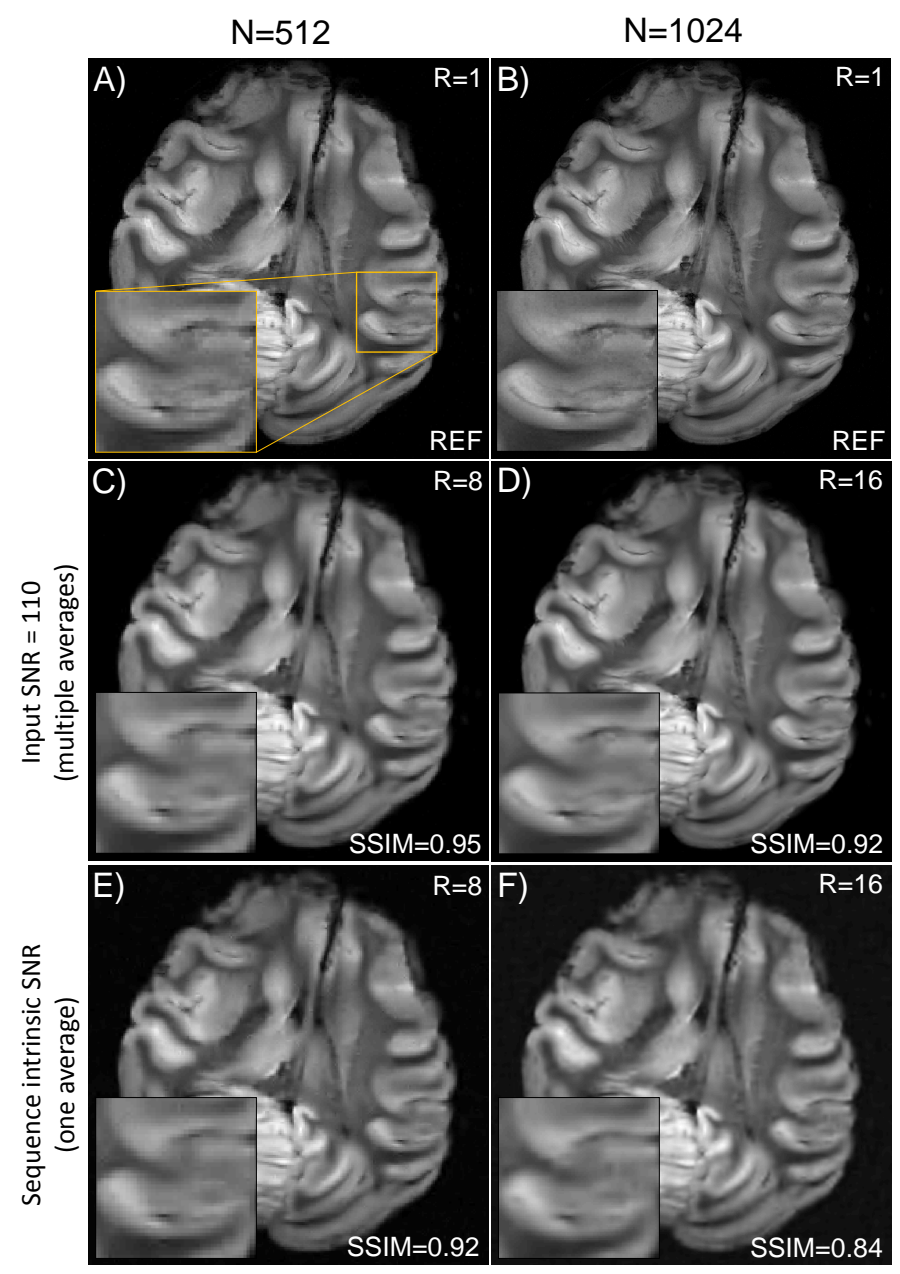

Figure 6: Results of retrospective iid-undersampling performed on experimental data acquired with a $T_{2}^{*}$-weighted GRE sequence (see sections III.C). Fully-sampled Cartesian reference images presenting an input SNR of 110 for $N=512$ (A) and $N=1024$ (B). A 2x-zoom displaying a folded pattern in the baboon cortex of visual interest has been added in the bottom left-hand corner for each image. For the intrinsic SNR of the considered acquisition (no average), reconstructions for images sizes $N=512$ (E) and $N=1024$ (F), which were undersampled respectively by a factor $R=8$ and $R=16$, are shown together with their SSIM scores calculated against their respective reference. In the case of an increased input SNR of 110 which was obtained by multiple averaging ( 82 and $15 \mathrm{NEX}$ for $N=1024$ and $N=512$ respectively), reconstructions for images sizes $N=512$ (C) and $N=1024$ (D), accelerated respectively by a factor $R=8$ and $R=16$ are displayed likewise. 

tice for this particular sequence to reach the targeted image quality $Q_{0}=0.9$. This $N$-SNR level line interestingly presents a maximum of 8 reached for an image size of $N=512$. More precisely, between $N=256$ and $N=512$, we observe that the $N$-SNR level line begins to deviate from the envelope of the are performing worse than iid sampling, with a difference of approximately 0.1 in the asymptotic SSIM scores for both experimental and simulated results. Nevertheless, as the input SNR falls to lowest values, the gap between the 


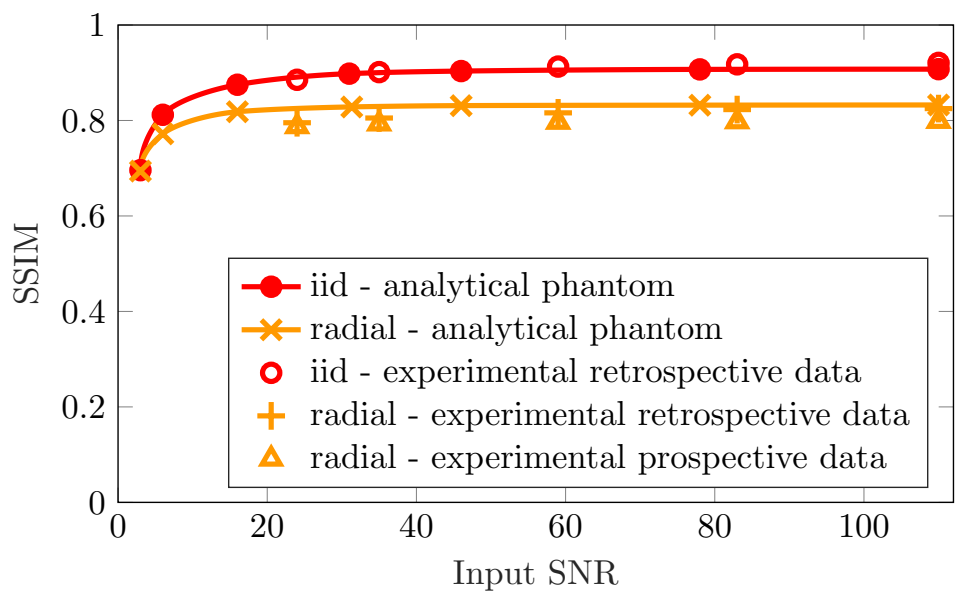

Figure 7: For an image size $N=1024$ and an acceleration factor of $R=15$, SSIM performance of iid (in red) and radial (in yellow) sampling schemes. Results obtained for the analytical phantom are represented by continuous lines while the experimental data on the ex vivo baboon brain are symbolized by discrete symbols. For radial sampling, prospectively acquired results are represented with triangles, while retrospective reconstructions on experimental data are displayed with cross signs.

two different sampling schemes shrinks to zero. Theses results illustrate how iid sampling schemes can produce near upper bounds to other 2D MR-feasible sampling trajectories such as radial readouts.

\section{Discussion}

In this empirical study, we showed how to quantitatively derive the maximum undersampling factor preserving a targeted image quality as a function of the image size and the input SNR. Hence, our results for $T_{2}^{*}$-weighted MRI provide quantitative guidelines on how to select subsampling factors and resolution in the framework of compressed sensing. On the one hand, our analysis quantitatively confirmed the benefits of going higher in resolution and is therefore in agreement with the presented theoretical results of CS (Section II - Fig. 1). By increasing the image size, larger acceleration factors can indeed be used while allowing to recover high-resolution details. Nevertheless, this enhanced performance at high resolution is in practice limited by the available SNR of the 
considered acquisition. We thus showed the existence of a minimum SNR that which is not always clearly stated in previous studies in CSMRI. This $R_{\max }$ function is theoretically defined over all possible combinations of image sizes and SNRs in the studied domain, but in practice remains confined to a more restricted area owing to the intrinsic SNR limitations of a particular MR acquibetween image size and SNR. In situations where the intrinsic SNR is limiting, it may be more favorable to use a smaller accelerating factor on a lower resolution scan for optimal results.

Regarding the practical utilization of this work, our experimental results appeared to be in good agreement with simulations performed on the analytical brain phantom, which corroborates the validity of our approach to derive a maximum undersampling factor for a given acquisition-reconstruction setup. Our work may thus aid the design of undersampled 3D acquisitions using CS and even 4D MRI, even though prospective performance of compressed MR acquisitions may be slightly lower than predicted due to unconsidered MR system imperfections (e.g., eddy currents). Moreover, our comparison with experimental radial sampling performance suggests that our results may be extended to $2 \mathrm{D}$ acquisitions insofar as they provide close upper bounds on the maximum undersampling factor which may be approached with spiral readouts [18]. Although in practice it is not always possible to acquire the fully-sampled Cartesian image to compute the available input SNR, MR physicists could estimate their acquisition intrinsic SNR by probing the noise and signal strength in the k-space directly.

Since this study assumes a particular acquisition and reconstruction pipeline, SSIM scores generated with another setup (e.g., parallel imaging and multichannel CSMRI reconstruction, see for instance [41, 42, 43]) may lead to slightly different quality scores. However, we expect the observed tendencies and dynamics to remain similar with other methods (e.g. changes in sparsifying dictionary or algorithm), with possibly minor up- or down-shifts of the SSIM curves. If 
the arbitrary choice of the SSIM quality threshold is not an issue itself since any user should be able to determine the image quality of interest against a chosen reference, some limitations of the SSIM index should be pointed out. First, the comparison of scores between different image sizes should be done carefully since the SSIM metric is not resolution-invariant: an image of size $N=1024$ presenting a SSIM of 0.92 may look better than an image of size $N=512$ presenting a SSIM of 0.95 , as can be observed on Fig. 6B)-C). The explanation lies in the fact that matching a reference of higher resolution is inherently more demanding. This issue can be bypassed by adjusting the desired image quality (i.e. the threshold) to the resolution via a threshold function $Q_{0}(N)$. Furthermore, the influence of the reference should also be mentioned as SSIM scores are likely to change with the input SNR of the reference notably. We evaluated the impact of this parameter in Appendix A and showed that this effect is of minor concern for the considered SNR. The structural characteristics of different images are also expected to influence the SSIM scores since this metric was designed to be sensitive to structural features. Nevertheless, our experimental results on a brain baboon showed SSIM scores very close to those of the analytical brain phantom despite significant structural differences between these two types of images (Fig. 6 and 7), which supports the consistency of the presented study. Although this study uses $T_{2}^{*}$ data, it is not unreasonable to expect the same behavior and quantitative estimations for other contrast such as $T_{2}$ and $T_{1}$, but this should

be confirmed with further experiments. Finally, the code and data used for this study are freely accessible on the website cosmic.cosmostat.org/code.

\section{Conclusion}

In the case of $T_{2}^{*}$-weighted MRI, our study empirically and quantitatively showed how the image quality measured with the SSIM index is affected by both input SNR and image size when Compressed Sensing alone is used to speed up MRI acquisition and how this information can be used to determine a maximum acceleration factor depending on the targeted image quality. On the one hand, 
our results confirmed the benefits of going higher in resolution since increasing the image size allows the use of larger acceleration factors. Nevertheless, this enhanced performance at high resolution is in practice limited by the available $\mathrm{SNR}$ in the considered acquisition. We showed the existence of a minimum SNR required to meet the expected image quality. Once this minimum SNR is reached, increasing the input SNR does not improve the image quality anymore. Most interestingly, we showed how to quantitatively derive the maximum undersampling factor $R_{\max }$ preserving a targeted image quality as a function of the image size and the input SNR. When the input SNR is high enough, typical $R_{\max }$ values were found to be 5, 8, 16 and 30 for image sizes 256, 512, 1024 and 2048 respectively for the considered $\ell^{1}$-based reconstruction. In practice, this

${ }_{440} R_{\max }$ function is however constrained by SNR limitations inherent to a particular MR acquisition and we demonstrated how our results enable to take this a priori knowledge into account. These results were corroborated by experiments performed on a 7 Tesla scanner both retrospectively and prospectively on radial data. They illustrate how the use of CS in acquisitions can be virtually limited by the available SNR when exploring very high resolution. Finally, our proposed SSIM-based method to determine the maximum acceleration factor for a particular acquisition can easily be adapted to any acquisition-reconstruction pipeline and may thus be of interest to other techniques such as parallel imaging.

\section{Acknowledgments}

The authors are thankful to Dr Patrick Martigne (Department of Radiobiology, IRBA, Brétigny-sur-Orge, France) for the preparation of the ex vivo baboon brain. The authors would also like to acknowledge the COSMIC project funded by CEA DRF-Impulsion.

\section{Appendix A. Influence of reference SNR on SSIM scores}

To evaluate the influence of the input SNR of the reference $\mathbf{x}$ on the scores $\operatorname{SSIM}(\widehat{\mathbf{x}}, \mathbf{x})$ scores, the reference SNR was varied in the computation of the SSIM 
for a fixed reconstruction $\widehat{\mathbf{x}}$ of the analytical brain phantom with iid sampling schemes. Two cases were tested and are presented in Fig. A.8 for (i) $N=512$, $R=5$ and an input SNR of 46 (black line) and (ii) $N=1024, R=10$ and an input SNR of 110 (orange line). Both curves present a stationary value as the input SNR of the reference increases. This graph shows that above an input SNR of approximately 100, the SNR of the reference no longer influences the value of SSIM scores. Noisier references do not match as well the reconstructed images probably because of the denoising effect of the nonlinear reconstructions. This result supports the use of a reference presenting an input SNR of 110 for the calculation of SSIM scores.

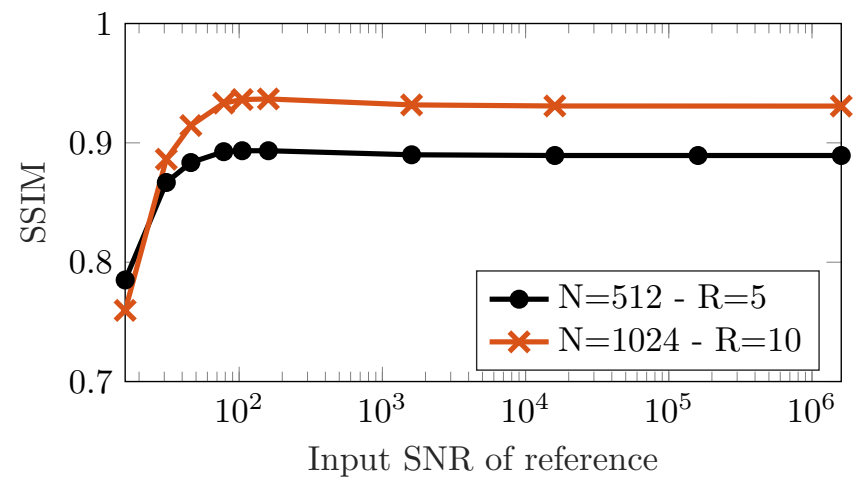

Figure A.8: Influence of input SNR of the reference on the SSIM scores calculated for fixed reconstructions in the case of a 5 -fold undersampled image size of $N=512$ for an input SNR of 46 (black line) and of a 10-fold undersampled image size of $N=1024$ for an input SNR of 110 (orange line). The logarithmic scale was used for the $\mathrm{x}$-axis.

\section{References}

[1] M. Lustig, D. Donoho, J. M. Pauly, Sparse MRI: The application of compressed sensing for rapid MR imaging, Magnetic Resonance in Medicine 58 (6) (2007) 1182-1195.

[2] M. Lustig, D. L. Donoho, J. M. Santos, J. M. Pauly, Compressed sensing MRI, IEEE Signal Processing Magazine 25 (2) (2008) 72-82. 
[3] Siemens Healthineers, Compressed sensing supplement, MAGNETOM Flash 2016 (66) (2016) 1-60.

[4] U. Gamper, P. Boesiger, S. Kozerke, Compressed sensing in dynamic mri, Magnetic resonance in medicine 59 (2) (2008) 365-373.

[5] H. Jung, K. Sung, K. S. Nayak, E. Y. Kim, J. C. Ye, k-t focuss: A general compressed sensing framework for high resolution dynamic mri, Magnetic Resonance in Medicine 61 (1) (2009) 103-116.

[6] O. Vardoulis, P. Monney, A. Bermano, A. Vaxman, C. Gotsman, J. Schwitter, M. Stuber, N. Stergiopulos, J. Schwitter, Single breath-hold 3d measurement of left atrial volume using compressed sensing cardiovascular magnetic resonance and a non-model-based reconstruction approach, Journal of Cardiovascular Magnetic Resonance 17 (1) (2015) 1.

[7] M. Usman, D. Atkinson, F. Odille, C. Kolbitsch, G. Vaillant, T. Schaeffter, P. G. Batchelor, C. Prieto, Motion corrected compressed sensing for freebreathing dynamic cardiac mri, Magnetic resonance in medicine 70 (2) (2013) 504-516.

[8] L. Feng, L. Axel, H. Chandarana, K. T. Block, D. K. Sodickson, R. Otazo, Xd-grasp: Golden-angle radial mri with reconstruction of extra motionstate dimensions using compressed sensing, Magnetic resonance in medicine 75 (2) (2016) 775-788.

[9] L. Feng, R. Grimm, K. T. Block, H. Chandarana, S. Kim, J. Xu, L. Axel, D. K. Sodickson, R. Otazo, Golden-angle radial sparse parallel mri: Combination of compressed sensing, parallel imaging, and golden-angle radial sampling for fast and flexible dynamic volumetric mri, Magnetic resonance in medicine 72 (3) (2014) 707-717.

[10] J. P. Haldar, D. Hernando, Z.-P. Liang, Compressed-sensing MRI with random encoding, IEEE Transactions on Medical Imaging 30 (4) (2011) 893-903. 
[11] G. Puy, J. P. Marques, R. Gruetter, J.-P. Thiran, D. Van De Ville, P. Vandergheynst, Y. Wiaux, Spread spectrum magnetic resonance imaging, IEEE transactions on medical imaging 31 (3) (2012) 586-598.

[12] B. Adcock, A. C. Hansen, C. Poon, B. Roman, Breaking the coherence barrier: A new theory for compressed sensing, arXiv preprint arXiv:1302.0561.

[13] B. Adcock, A. C. Hansen, Generalized sampling and infinite-dimensional compressed sensing, Foundations of Computational Mathematics (2015) $1-61$.

[14] C. Boyer, P. Weiss, J. Bigot, An algorithm for variable density sampling with block-constrained acquisition, SIAM Journal on Imaging Sciences 7 (2) (2014) 1080-1107.

[15] J. Bigot, C. Boyer, P. Weiss, An analysis of block sampling strategies in compressed sensing, IEEE Transactions on Information Theory 62 (4) (2016) 2125-2139.

515 [16] N. Chauffert, P. Ciuciu, J. Kahn, P. Weiss, Variable density sampling with continuous trajectories. Application to MRI, SIAM Journal on Imaging Sciences 7 (4) (2014) 1962-1992.

[17] N. Chauffert, P. Weiss, J. Kahn, P. Ciuciu, A projection algorithm for gradient waveforms design in Magnetic Resonance Imaging, IEEE Transactions on Medical Imaging 35 (9) (2016) 2026-2039.

[18] C. Boyer, N. Chauffert, P. Ciuciu, J. Kahn, P. Weiss, On the generation of sampling schemes for Magnetic Resonance Imaging, SIAM Journal on Imaging Sciences 9 (4) (2016) 2039-2072.

[19] J. Aelterman, H. Q. Luong, B. Goossens, A. Pižurica, W. Philips, Aug525 mented Lagrangian based reconstruction of non-uniformly sub-nyquist sampled MRI data, Signal Processing 91 (12) (2011) 2731-2742. 
[20] M. Guerquin-Kern, M. Haberlin, K. P. Pruessmann, M. Unser, A fast wavelet-based reconstruction method for magnetic resonance imaging, IEEE transactions on medical imaging 30 (9) (2011) 1649-1660.

[27] F. Krahmer, R. Ward, Stable and robust sampling strategies for compressive imaging, IEEE transactions on image processing 23 (2) (2014) 612-622.

[28] C. Boyer, J. Bigot, P. Weiss, Compressed sensing with structured sparsity and structured acquisition, Applied and Computational Harmonic Analy-

[29] T. Zhang, S. Chowdhury, M. Lustig, R. A. Barth, M. T. Alley, T. Grafendorfer, P. D. Calderon, F. J. Robb, J. M. Pauly, S. S. Vasanawala, Clinical performance of contrast enhanced abdominal pediatric mri with 
fast combined parallel imaging compressed sensing reconstruction, Journal of Magnetic Resonance Imaging 40 (1) (2014) 13-25.

[30] M. Guerquin-Kern, L. Lejeune, K. P. Pruessmann, M. Unser, Realistic analytical phantoms for parallel magnetic resonance imaging, IEEE Transactions on Medical Imaging 31 (3) (2012) 626-636.

[31] N. Chauffert, P. Ciuciu, P. Weiss, Variable density compressed sensing in MRI. Theoretical vs. heuristic sampling strategies, in: Proc. of 10th IEEE ISBI conference, San Francisco, USA, 2013, pp. 298-301.

[32] S. Winkelmann, T. Schaeffter, T. Koehler, H. Eggers, O. Doessel, An optimal radial profile order based on the Golden Ratio for time-resolved MRI, IEEE Transactions on Medical Imaging 26 (1) (2007) 68-76.

[33] J. Keiner, S. Kunis, D. Potts, Using NFFT 3 - a software library for various nonequispaced fast fourier transforms, ACM Transactions on Mathematical Software (TOMS) 36 (4) (2009) 19.

[34] A. Beck, M. Teboulle, A fast iterative shrinkage-thresholding algorithm for linear inverse problems, SIAM journal on imaging sciences 2 (1) (2009) $183-202$.

[35] Z. Wang, A. C. Bovik, H. R. Sheikh, E. P. Simoncelli, Image quality assessment: from error visibility to structural similarity, IEEE Transactions on Image Processing 13 (4) (2004) 600-612.

[36] K. G. Hollingsworth, D. M. Higgins, M. McCallum, L. Ward, A. Coombs, V. Straub, Investigating the quantitative fidelity of prospectively undersampled chemical shift imaging in muscular dystrophy with compressed sensing and parallel imaging reconstruction, Magnetic Resonance in Medicine 72 (6) (2014) 1610-1619.

[37] L. W. Mann, D. M. Higgins, C. N. Peters, S. Cassidy, K. K. Hodson,

A. Coombs, R. Taylor, K. G. Hollingsworth, Accelerating MR imaging 
liver steatosis measurement using combined compressed sensing and parallel imaging: a quantitative evaluation, Radiology 278 (1) (2015) 247-256.

[38] O. Jeromin, M. S. Pattichis, V. D. Calhoun, Optimal compressed sensing reconstructions of fMRI using $2 \mathrm{D}$ deterministic and stochastic sampling geometries, Biomedical engineering online 11 (1) (2012) 1.

[39] M. Zanforlin, D. Munaretto, A. Zanella, M. Zorzi, SSIM-based video admission control and resource allocation algorithms, in: Modeling and Optimization in Mobile, Ad Hoc, and Wireless Networks (WiOpt), 2014 12th International Symposium on, IEEE, 2014, pp. 656-661.

[40] C. Lazarus, P. Weiss, N. Chauffert, F. Mauconduit, A. Vignaud, P. Ciuciu, Sparkling: Novel non-cartesian sampling schemes for accelerated $2 \mathrm{~d}$ anatomical imaging at $7 \mathrm{t}$ using compressed sensing, in: Proceedings 25th Scientific Meeting, International Society for Magnetic Resonance in Medicine, Honolulu, 2017.

[41] D. Liang, B. Liu, J. Wang, L. Ying, Accelerating SENSE using compressed sensing 62 (6) (2009) 1574-1584.

[42] L. Chaari, J.-C. Pesquet, A. Benazza-Benyahia, P. Ciuciu, A wavelet-based regularized reconstruction algorithm for SENSE parallel MRI with applications to neuroimaging 15 (2) (2011) 185-201.

[43] I. Y. Chun, B. Adcock, T. M. Talavage, Efficient compressed sensing SENSE pMRI reconstruction with joint sparsity promotion 35 (1) (2016) 354-368. doi:10.1109/TMI.2015.2474383. 